\title{
Cacher, festive et végétarienne
}

\author{
La nouvelle cuisine des fêtes juives \\ Kosher and Vegan \\ Jewish Holidays' New Forms of Cooking
}

Sophie Nizard

Résumé : Alors que la viande et les produits carnés sont fortement valorisés par la culture culinaire juive traditionnelle, on assiste depuis une dizaine d'années à un développement spectaculaire des pratiques végétariennes ou véganes en milieu juif, en particulier aux Etats-Unis et en Israël. Cette nouvelle manière de manger est justifiée par les mangeurs eux-mêmes et par nombre de rabbins comme prenant sa source dans la Bible hébraïque et dans la littérature rabbinique. En effet, l'interdit de causer de la souffrance aux animaux apparaît comme un principe fort des textes de la tradition juive. Ces changements sont repérables du fait de l'augmentation de l'offre en Israël (apparition de produits green et certifiés " sans matière animale » dans les supermarchés, multiplication de restaurants vegan friendly). Les discours de femmes juives sur leurs pratiques alimentaires et culinaires, recueillis au début de l'année 2020, viennent illustrer ces changements qui s'avèrent progressifs et sont rarement le résultat de choix idéologiques radicaux.

Mots-clefs : Cacher, cuisine végétarienne, Israël, judaïsme, souffrance animale, végan, viande

Abstract: While meat food is valued socially and symbolically as a part of
traditional Jewish culinary culture, vegetarianism and veganism among Jews
increased quite spectaculary over the past decade, especially in the USA and in
Israel. According to rabbis and to interviewees themselves this new way of eating
rooted in the Hebrew Bible and in the rabbinic literature. Indeed causing any
cruelty or suffering to animals is prohibited according to these sacred sources; this
is an absolute principle. Such changes are having effects on the increment of the 
products that are certified "green" and on the increase of vegan friendly restaurants in Israel. The narrative of Jewish women about their food and culinary practices shows those ongoing changes which are often not the result of ideological radical choices.

Keywords: animal suffering, Israel, Judaism, Kosher, meat food, vegan, vegetarian cooking

Le calendrier juif, à la fois lunaire et solaire, est ponctué par des fêtes religieuses dont le cycle saisonnier correspond à celui des activités agricoles. Les trois grandes fêtes annuelles donnaient lieu, dans l'Antiquité juive, à un pèlerinage à Jérusalem ; Souccoth (Les Cabanes) est généralement célébrée en septembre/octobre, Pessah (la Pâque) en mars/avril et Shavouoth (la Pentecôte) en mai/juin. D’autres fêtes sont célébrées annuellement comme Rosh hashana (le jour de l'An) et Yom Kippour (le jour du Pardon) au début de l'automne, Hannouka, la fête des Lumières, autour du solstice d'hiver et Pourim (les Sorts) en février ou mars. Mais la fête principale de ce calendrier est sans aucun doute le Shabbat, septième jour de la semaine, temps saint par excellence ; il fait mémoire de la création du monde en six jours et du repos du créateur le septième jour. Au-delà d'être un jour hebdomadaire de repos et de prière, le Shabbat est avant tout un moment de convivialité et de commensalité, tant les trois repas prescrits de ce jour sont importants dans la tradition religieuse. De la même manière, chaque fête juive fait mémoire d'événements marquants de l'histoire religieuse et est souvent caractérisée par des nourritures rituelles. Ainsi, le pain azyme et les herbes amères de la Pâque rappellent la sortie d'Égypte, la pomme trempée dans du miel au soir du Nouvel An augure d'une année douce et heureuse, les laitages de Shavouoth symbolisent le don de la Torah $^{1} .$. Tous ces repas de fête et de Shabbat s'ouvrent par une bénédiction sur le vin (sanctification ou kiddoush), puis sur le pain (motsi) et sont clôturés par des actions de grâce. La prière fait partie intégrante de tout repas, il s'agit à chaque rencontre avec les nourritures à la disposition des hommes d'en rappeler l'origine et de remercier leur créateur. Ainsi, la cuisine scande le temps festif et imprime les imaginaires du rythme, de l'ambiance, des odeurs et des saveurs caractéristiques des moments de fête. $\mathrm{Si}$, pour certaines familles, le religieux en lui-même n'est pas toujours structurant, les identités sont forgées par ces nourritures dont la mémoire sensitive et affective remonte à l'enfance. Ainsi Edgar Morin, revenant sur son identité juive, écrit :

Ce qui ressort de tout cela, c'est une éducation dans un vide religieux, n'ayant pour seule nourriture cultuelle vraiment spécifique qu'une culture alimentaire : gratin d'aubergine, pastels, borekas ${ }^{2}$, cuisine à base d'huile d'olive ; pour moi, c'est mon identité la plus profonde et chérie. Le plat que je préfère au monde, c'est le gratin d'aubergine. (Morin 1984) 
Dans le monde ashkénaze (celui d'Europe Centrale et de l'Est) comme dans le monde sépharade (issu de la Péninsule Ibérique-Afrique du Nord, Turquie, Grèce...) ou moyen-oriental, les nourritures carnées sont la marque des repas festifs. Si nous reprenons le concept de "plat-totem ${ }^{3}$ », chaque groupe culturel s'autoreprésente ou est vu comme porteur d'un plat qui semble le caractériser. Le gefilte fish (carpe farcie) emblématique de la cuisine juive d'Europe de l'Est, le couscous accompagné de boulettes de viande des Juifs de Tunisie, les tajines et pastels marocains, les borekas grecs et, partout, le plat du Shabbat, conçu pour rester sur un feu couvert du vendredi soir jusquau déjeuner du samedi midi : qu'ils prennent le nom de tchoulent ici, de bkeila, de dafina ou de tfina là, tous ces plats ou presque sont confectionnés à partir de chair animale. La dafina, ce ragoût de bœuf et de céréales (généralement de blé ou d’orge), de riz, de légumineuses (haricots secs, pois-chiches), de pommes de terre entières et d'œufs cuits dans leur coquille est typique du Shabbat marocain. Mijotés durant plus de douze heures, les aliments prennent une jolie couleur brune dans la marmite, parfois emmitouflée sous des couvertures pour éviter toute déperdition de chaleur. En effet, l'interdit d'allumer un feu et de cuire des aliments durant le Shabbat ${ }^{4}$ donne lieu, ici et là, à l'élaboration de mets spécifiques à ce jour, préparés et cuits à l'avance, aptes à rester des heures sur un feu très doux (Bahloul 1981). Ce feu (aujourd'hui une plaque chauffante électrique), allumé le vendredi soir avant le coucher du soleil, n'est pas entretenu pendant les vingt-cinq heures que dure la fête. Ces prescriptions donnent une couleur et un goût particuliers aux mets du Shabbat midi souvent confits, mijotés, fondants, tant leur cuisson a été lente et continue. Les cuisines, le vendredi, jour où ces mets sont préparés et cuisinés, sont emplies des odeurs de pain, de gâteaux, de viandes rôties, de poisson, d'épices et de tous les mets élaborés qui contribueront à « honorer le Shabbat». C'est cela qui conduit ceux qui décrivent l'atmosphère des préparations de fêtes à évoquer les odeurs de Shabbat:

Le Shabbat chez ma mère, c'est les bonnes odeurs, les choses préparées à l'avance, quelque chose de plus que l'ordinaire. En tout cas, moi, quelque chose que je garde toujours à l'esprit, c'est la fête juive, c'est-à-dire la transformation de la maison et des individus parce que c'est la fête, parce que c'est Shabbat... C'est associé dans ma tête à des odeurs, à des couleurs, à des odeurs du vendredi soir, particulières par rapport aux autres soirs de la semaine, des odeurs à la fois de couscous et de gâteaux, je ne sais pas, c'est une odeur confortable, reposante, une odeur chaleureuse... Comme un cocon, une odeur de matrice. Et ça, j'en suis reconnaissante, pas forcément à ma famille, parce quelle ne faisait que reproduire des traditions...

Pourtant, ces plats traditionnels du Shabbat ont été remplacés au fil du temps par des mets moins élaborés, moins riches, inspirés de la cuisine continentale; dans les familles pratiquantes, ceux-ci sont posés une ou deux heures avant le repas du samedi midi sur une plaque chauffante, branchée depuis la veille au soir, afin d'atteindre une température convenable : poulet ou autres 
viandes rôties, accompagnées de pommes de terre au four, de riz ou de blé, gratins de légumes... Dans les milieux séfarades, lors des repas de Shabbat ou de fêtes, pour le dîner comme pour le déjeuner du lendemain midi, la table est remplie d'un grand assortiment de salades cuites ou de crudités; cette kémia et sa diversité sont gage d'abondance et de réception réussie. Sur les tables marocaines, on trouve souvent un plat de poisson en sauce rouge épicée en guise d'entrée ; sur les tables tunisiennes, outre l'eau de vie de figue qui l'accompagne, l'apéritif comprend immanquablement de la boutargue (œufs de mulet salés et séchés) coupée en fines tranches et citronnée, des bricks, des fricassés ${ }^{6}$, de la minina ${ }^{7}$... Les repas des fêtes juives, quelle que soit la provenance des mangeurs, sont ainsi presque toujours confectionnés à base de produits d'origine animale. Même les desserts ou les gâteaux, s'ils ne contiennent souvent ni lait ni beurre ${ }^{8}$, sont le plus souvent confectionnés à base d'œufs.

Pourtant, Anna raconte à propos des échanges de pâtisseries propres à la fête de Pourim ${ }^{9}$ comment elle a dû confectionner des cookies sans œufs ni beurre, mais à base de crème de sésame (tehina) pour les offrir à ses amis végans, étudiant avec elle à la Faculté d'agriculture de Rehovot (ville universitaire au sud de Tel Aviv) ${ }^{10}$.

La simplification des préparations culinaires par rapport à la génération précédente apparaît dans ce propos :

Ma mère préparait son ragoût... le vendredi matin, elle faisait toute la cuisine pour vendredi et samedi. Elle n'allumait pas le feu pour réchauffer. Moi, j’allume le feu; si j'ai le lait à faire réchauffer le matin pour les enfants, je le fais. Je ne fais pas le pain, je vais l'acheter (...). En général, je fais un couscous, mais sans boulettes, parce que c'est compliqué, et je prépare les salades à peu près comme le fait ma belle-mère... (Nizard $1997: 136$ ).

Ces traditions culinaires carnées sont renforcées par des sources textuelles. A propos de la fête des Cabanes, le Deutéronome (XVI ;14) enjoint : " Tu te réjouiras lors de ta fête ». Pour les sages du Talmud commentant ce verset ${ }^{11}$ et s'appuyant sur les Psaumes ${ }^{12}$, celui qui se réjouit est celui qui consomme du vin. Cependant, pour l'un d'eux, Rabbi Yehuda Ben Betera, à l'époque où le Temple ${ }^{13}$ existait, ce qui réjouissait l'homme, c'était la consommation de viande, mais depuis sa destruction, ça ne peut être que le vin. Bien plus tard, Maïmonide, médecin, grand commentateur et décisionnaire du Moyen-Âge, ajoutera " Il n'y a pas de joie sans viande ni vin ».

Citant cet adage, une jeune femme interrogée sur sa réticence à manger de la viande déclare : "A Pessah, j'aime manger de l'agneau, ça fait vraiment partie de nos habitudes, de notre culture ; mais le Shabbat, je ne mange plus de viande. On essaye de manger des choses qui nous font plaisir, et surtout je compense en buvant du vin! $»^{14}$

Dès lors, est-il possible que les Juifs soucieux de ces traditions religieuses suivent en même temps un régime végétarien ou végan ? Comment préparer un Shabbat sans viande ni poisson, sans gâteau, sans ces mets traditionnels 
transmis de génération en génération ? Tout anthropologue de l'alimentation sait que les modes de préparation évoluent dans le temps, au sein des groupes, des familles et même pour un même individu en fonction de son savoir culinaire, de sa conscience des normes alimentaires anciennes et nouvelles et de leur confrontation, de sa santé et de celle de ses proches, des ingrédients dont il dispose, des mobilités géographiques et des migrations, donnant lieu à des métissages alimentaires (Kanafani-Zahar, Mathieu et Nizard 2007).

Lévolution des connaissances en matière d'alimentation et l'impact de celle-ci sur la santé ont d'ores et déjà profondément changé les modes de préparation des aliments et la composition des mets. De la même manière, les temps consacrés à la préparation des nourritures à l'intérieur des foyers ont sensiblement diminué avec le travail des femmes, l'externalisation de nombreuses préparations auparavant effectuées dans les cuisines et l'évolution des techniques culinaires et de conservation (usage des robots ménagers, des réfrigérateurs, des congélateurs, du micro-ondes, des cocottes minutes...).

Qui plus est, après des années d'augmentation, la diminution de la consommation de nourritures carnées au cours des dix dernières années est significative (Bouanchaud 2018) ; certains réduisent leur consommation de viande, d'autres, moins nombreux, deviennent flexitariens ${ }^{15}$, végétariens ou végans, pour des raisons de santé, de respect de l'environnement ou de souci envers les animaux.

L'interdit absolu de "causer une souffrance quelconque aux animaux » (interdit du tza’ar ba’alei hayyim) trouve sa source dans la Bible hébraïque ou Torah (Loi écrite) et est explicité dans le Talmud. Or dans le contexte contemporain d'élevage intensif et d'abattage de masse, il est de plus en plus difficile de garantir ce principe ${ }^{16}$. C'est pourquoi certains rabbins, penseurs ou commentateurs contemporains des textes de la tradition juive, notamment aux États-Unis et en Israël, tentent de repenser les lois alimentaires juives (lois de cacherout $^{17}$ ) et de les orienter vers le végétarisme, voire le véganisme ${ }^{18}$. Ainsi le Rabbin Arthur Green (Professeur au Hebrew College de Boston), dans un article intitulé "Vegetarianism : An Alternative Kashrut " (Green s.d.), rappelle que la tradition juive, pour des raisons morales et spirituelles, récuse la violence et la cruauté, et réprouve par conséquent le sang versé des bêtes et la consommation carnée. En allant plus loin, il affirme que les Juifs doivent aujourd'hui se sentir, plus que tous les autres pour des raisons morales et religieuses, concernés par le souci de partager les ressources limitées de la terre ; ils devraient en outre s'engager à suivre un mode de vie plus sain et reconnaître que, somme toute, les humains sont une espèce plutôt végétarienne. L'étape suivante devrait-être, selon Green, de devenir résolument végétariens. Enfin, ultime argument du rabbin Green, l'histoire récente des Juifs pendant la Seconde Guerre mondiale devrait les prémunir contre les traitements inacceptables infligés aujourd'hui aux animaux, comparant quasi-explicitement les abattoirs modernes aux camps de la mort ${ }^{19}$. Yosi Fargeon (Institut Herzog, Israël) montre, versets de la Bible à l'appui, que la tradition juive promeut, dès 
l'origine, une alimentation végétarienne. Pour lui, la création par Dieu des animaux comestibles uniquement dans le but de permettre à l'homme d'en consommer la viande est un mythe (Fargeon 2013). Dans un autre registre, Assa Keissar, prédicateur religieux du végétarisme, promet aux mangeurs de viande " mesure pour mesure $»^{20}$ pour leur manque de compassion à l'égard des animaux (Keissar 2019).

Ces penseurs contemporains du judaïsme ont des précurseurs. Ainsi, le rabbin Shlomo Ephraim Luntschitz (Prague, fin du seizième siècle) ou le rabbin Abraham Isaac Kook (père du sionisme religieux et premier Grand rabbin ashkénaze de Palestine dès 1921, c'est-à-dire à l'époque du Mandat britannique) pensaient que la complexité des lois alimentaires et l'éthique juives devaient décourager de manger de la viande. Car la cacherout, en plus d'être un ensemble extrêmement rigoureux de prescriptions et d'interdits sur les aliments eux-mêmes et leur composition, leur mode de préparation et de cuisson, est aussi et surtout une éthique vis-à-vis du monde : vis-à-vis des autres humains et vis-à-vis du reste de la création, la planète, les végétaux et les animaux. Adam, le premier homme, avait reçu le commandement, dans le jardin d'Eden, de travailler et de préserver la terre (Gen. II ; 15). Ce commandement adressé à l'humain résonne fortement pour notre pensée contemporaine travaillée par le souci écologique. Il est repris par les écologistes juifs comme une injonction à préserver l'environnement (Abramowitz 2018). Dans le récit de la Genèse, Dieu, qui fait résider Adam et Eve dans l'Eden, met à leur disposition " tous les arbres du jardin ", mais de consommation animale, il n'en est pas question, pas encore... Le paradis primitif était donc végétarien. À la deuxième génération, celle des fils du couple originel, la Bible évoque un sacrifice animal offert par le berger Abel. Mais ce sacrifice, agréé par Dieu, excitera la jalousie de Caïn et conduira au premier fratricide. C'est seulement après le déluge qui, paradoxalement, voit le sauvetage par Noé des animaux dans l'arche, que l'humanité devient carnivore, comme si l'idéal végétarien de la création avait cédé face à la violence des hommes, comme si la consommation de viande était une concession temporaire. Mais cette autorisation est aussitôt assortie d'une interdiction : celle de la consommation de sang, car, indique le texte, «le sang c'est l'âme » (Gen. IX ; 2-4) et plus loin dans la Bible, "lâme de la chair est dans le sang ( Lev. XVII ; 11). Principe de vie par excellence, le sang, siège de l'âme ou âme elle-même, ne peut être consommé. Par une torsion discursive, la chair animale désormais comestible est dissociée du sang, porteur de vie ${ }^{21}$.

La plupart des interdits alimentaires de la tradition juive concerne les animaux eux-mêmes (certaines espèces sont proscrites), leur mode d'abattage, leur préparation et leur consommation. Aussi un régime végétarien (et $a$ fortiori végan) est cacher par essence ${ }^{22}$. En effet, les lois de cacherout classent de fait les aliments en trois catégories : les aliments carnés (à base de viande issue de mammifères ou de volailles permis à la consommation), les aliments contenant des laitages (lait, beurre, crème, fromages, issus de bovins ou ovins), 
et les aliments qui ne contiennent ni produit carné ni laitage, nommés aliments parvé (autrement dit neutres). Les poissons (s'ils sont cacher-c'est-à-dire pourvus d'écailles et de nageoires), les œufs et tous les végétaux font partie de cette troisième catégorie. Les deux premières catégories d'aliments (carnés et lactés) ne peuvent être préparés ensemble, en vertu de l'interdit biblique « Tu ne cuiras pas le chevreau dans le lait de sa mère ", ni même être consommés au cours d'un même repas (Nizard 2007 b). Pas d'escalope normande, pas de crème brulée après un repas carné, pas même de fromage confectionné à l'aide de présure animale (caillette) au cours d'un repas cacher. Par contre les aliments parvé peuvent être indifféremment consommés avec des aliments de l'une ou de l'autre catégorie ${ }^{23}$. Ainsi les desserts traditionnels de la cuisine juive sont souvent parvé (en l'occurrence sans laitage) pour pouvoir être consommés après un repas carné. Pour ces raisons, les restaurants cacher sont soit carnés, soit lactés, afin d'éviter tout mélange fortuit entre les deux types d'aliments. Dans la sphère domestique, les cuisines sont organisées de telle sorte que les espaces consacrés à la préparation des plats à base de laitages et des nourritures à base de viande ou de volaille sont strictement séparés. Dans les familles pratiquantes, dans la mesure du possible, il y aura deux éviers et des vaisselles différenciées afin d'éviter toute interférence entre ces deux types d'aliments incompatibles. De ce fait, il est évident que des personnes mangeant strictement cacher pourraient se simplifier la vie en excluant totalement de leur alimentation un des deux types d'aliments. Aussi, un régime végétarien domestique permettrait une simplification des règles de séparation : une seule vaisselle, un seul jeu de couverts, une seule batterie de casseroles : en bref, pas d'interférence possible entre laitages et produits carnés si ces derniers sont exclus.

De fait, tant pour des raisons pratiques qu'idéologiques ou éthiques, les Juifs contemporains, en particulier les plus pratiquants d'entre eux, seraient plutôt enclins à adopter un régime végétarien, voire végan (Krief 2019; Nizard 2020). Certes, la cuisine traditionnelle des fêtes juives est principalement carnée, mais parmi les générations les plus jeunes, nombreux sont ceux qui recomposent leur mode d'alimentation pour concilier pratiques religieuses et évitement des produits carnés, voire de tout produit animal.

Dès lors comment célébrer le Shabbat et les fêtes sans viande ni poisson ? Ce serait comme célébrer Thanksgiving sans $\operatorname{dinde}^{24}$, Noël sans chapon ou le jour de l'An sans foie gras, l'Ayd sans mouton...

Pourtant, les tendances que l'on observe dans l'ensemble des pays occidentaux (baisse de la consommation de viande, attention au corps par des pratiques alimentaires faisant une plus grande part aux végétaux-céréales, fruits et légumes, légumineuses) semblent encore plus présentes en Israël. Tel Aviv a été classée comme la capitale mondiale du végan ou en tout cas comme une ville « vegan friendly », avec ses 400 restaurants proposant des menus végans. D'ailleurs, le falafel, street food typiquement israélien ${ }^{25}$ fait de pain pita fourré de crudités et de boulettes de pois-chiche frites recouverts d'une sauce tahini, 
est végan par essence. Des échoppes en proposent à tous les coins de rue dans les villes israéliennes pour quelques shekels.

Au sein même de l'armée israélienne, le nombre de végans a été multiplié par 20 ces dernières années, portant à 1 sur 18 le nombre de soldats se déclarant végétaliens fin 2018 (Douillet 2018). Cette proportion d'environ 5 pour cent est sans doute représentative du nombre de végans dans la population israélienne globale. Mais si l'on y ajoute les végétariens, ce pourcentage atteindrait 10 à 15 pour cent de la population israélienne (Leban 2017), pourcentage bien supérieur aux estimations du nombre relatif de végétariens en France, en Allemagne ou au Royaume-Unis par exemple ${ }^{26}$.

Des livres de cuisine juive végane ou cacher végane sont publiés régulièrement. Par exemple, The Jewish Food Hero Cookbook regroupant « 50 recettes simples à base de plantes pour vos plats de fête " (Alfond 2019) alterne des recettes véganes et des récits autour de figures féminines de la Bible, accompagnés de légendes qui y sont attachées, et se propose de «nourrir l'âme le corps et l'esprit ». Le livre Plenty du chef israélien Yotam Ottolenghi (2015) propose 120 recettes végétariennes inspirées du répertoire culinaire méditerranéen et magnifiquement illustrées (See Figure 1).

Le groupe Facebook Vegan Tel Aviv (2020) est consacré aux questions que se posent les végans en Israël. Ceux-ci partagent leurs idées, leurs recettes et les photos de plats qu'ils ont préparés ; ici, une jeune femme donne la recette d'une crème de noix de cajou pour remplacer la sauce tahini faite à partir de


Figure 1 : Des livres de cuisine juive végane. Source : https://jewish-veg.myshopify.com/ collections/books/products/jewish-food-hero-cookbook; https:/www.booklarder.com/ books/info/plenty-vibrant-vegetable-recipes-from-londons-ottolenghi 


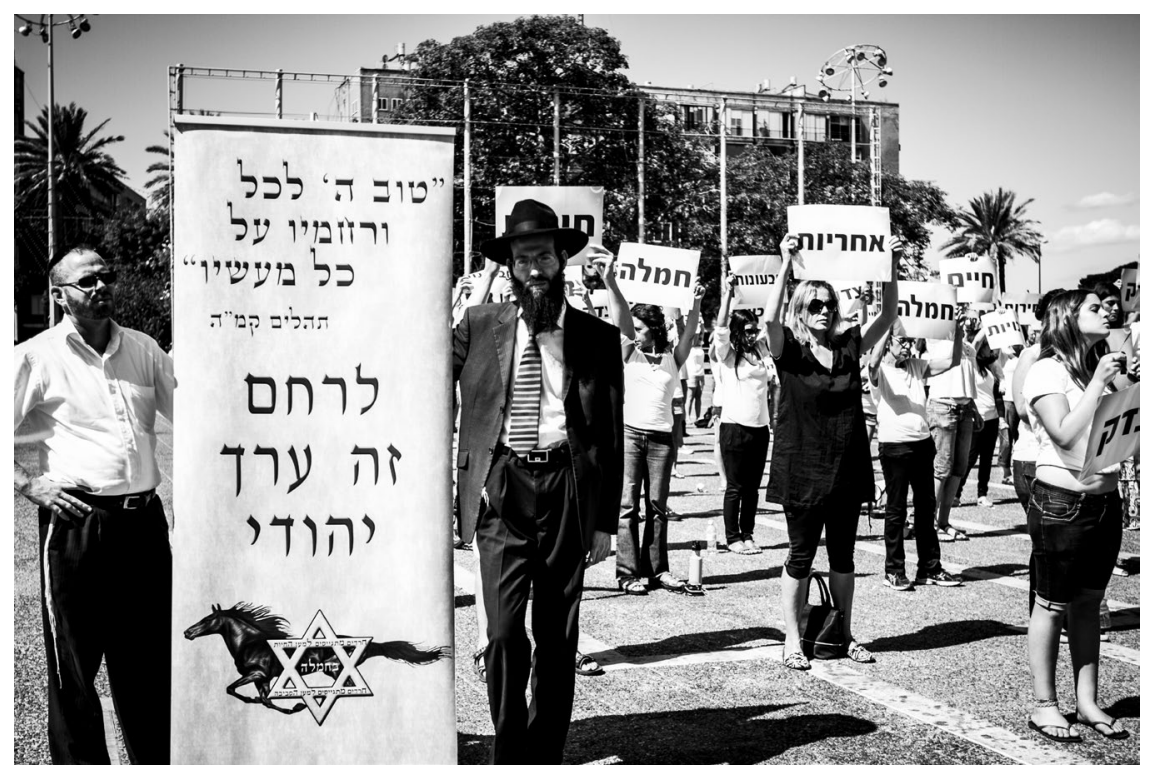

Figure 2 : Manifestation de militants de l'association Behemla en 2013, association luttant pour la cause animale. Behemla signifie en hébreu " avec compassion » ou " avec pitié ». La pancarte principale reprend le psaume 145 verset 9 "L'Éternel est bon pour tous, sa pitié sétend à toutes ses créatures ». Sur les pancartes en arrièreplan, on peut lire «pitié » ou " compassion ». Source : https://jewpop.com/opinions/ tous-les-juifs-devraient-etre-vegetariens/

graines (de sésame) que certains s'interdisent durant les huit jours de la Pâque : «Last year was the first time I made Passover as a Vegan. I was a little freaked, honestly. Tahini is made from sesame seeds, so that makes it a no-no. »

Des enseignes de supermarchés israéliens proposent des gammes «bio », « vegan », " sans gluten », comme la gamme Green de l'enseigne Shufersal, qui se présente comme une gamme soucieuse de "santé et nature ", ou la chaîne de boutiques Anise qui se définit comme " nature store ». La plupart des produits proposés sont labélisés à la fois comme organi, c'est-à-dire bio, et comme cacher, avec souvent plusieurs certifications religieuses pour un même produit, afin de s'adapter aux diverses tendances du paysage religieux israélien. Des substituts de viande sont particulièrement présents dans ces gammes, comme le tofu, le seitan, ou les schnitzels végétariens.

Des militants très impliqués dans la défense des animaux vont parfois jusquà utiliser la violence pour faire entendre leur voix dans le pays, comme Sasha Boojor ou Tal Gilboa (Leban 2017) (See Figure 2).

Mais la plupart des végétariens ne sont pas des militants, ni même des personnes très marquées par une idéologie végane, mais plutôt des flexitariens (mangeurs occasionnels de viande) qui justifient diversement leurs pratiques 
évoluant vers le végétarisme ou le véganisme. Écoutons ici certains de leurs propos :

J'aime manger, déclare Marina, j'aime la bonne chère, mais le problème c'est que je n'aime pas la chair! On peut remplacer la viande par autre chose. Hier, par exemple, j'ai fait revenir des choux pak choï dans du beurre, on peut aussi utiliser des aubergines, ça remplace bien la viande avec comme accompagnement d'autres légumes, des céréales ou même des crêpes. En fait, j’aime le goût de la viande, mais quand j'en mange, je sens le vivant, je le ressens clairement et ça me dégoûte. Je me souviens du temps où j'étais étudiante, il y avait, dans le labo où je préparais mon master, un végan religieux qui d’ailleurs était nutritionniste. Je lui avais dit : “Tu ne crois pas quon a assez de contraintes comme ça en mangeant cacher, pourquoi en rajouter ?" Maintenant, je le comprends. ${ }^{27}$

Ou encore Léa, qui dit avoir commencé à ne plus consommer de viande depuis six ou sept ans, car son mari ne voulait plus en manger, à cause de la maltraitance faite aux animaux. "Je te suis ", lui avait-elle affirmé. Depuis, le couple ne consomme plus de poulet, ni de viande, mais continue de manger œufs et poisson.

Ce n'est pas logique mais... Lui a aussi arrêté le poisson qui, moi, ne me dérange pas. En fait, c'est une manière de lutter contre le système, même si c'est une goutte d'eau dans la mer, je ne veux pas faire partie de ça. Surtout en ce moment, on ne peut pas ne pas réagir. On savait que ça allait venir, la façon dont on vit est beaucoup trop industrialisée. Les gens mangent n'importe quoi ! Il faut quon réfléchisse, même au-delà de la nourriture. Moi, c'est 100 pour cent à cause de la souffrance animale, mais quand les enfants viennent à la maison pour le Shabbat, ça m’arrive de cuisiner du poulet khofesh (élevé en plein air).

Et de continuer sur les effets bénéfiques de ce changement de régime : "On se sentait beaucoup mieux, notamment le vendredi soir, plus légers, plus d'énergie. On mange beaucoup plus de légumes, de légumineuses... Je mange moins, du coup ${ }^{28}$.

Les arguments de ces végétariens ou flexitariens relèvent de l'attention à leur santé, d'un réel souci pour les animaux, parfois du dégoût que leur inspire l'idée même de manger de la chair animale (See Figure 3).

Dans tous les cas, ils évoquent le temps important consacré à la préparation de repas à base de légumes qu'il faut laver et éplucher, l'imagination pour adapter les recettes traditionnelles ou en inventer de nouvelles, le budget nécessaire pour ceux qui en outre achètent des produits bios ou, pour ceux qui en consomment, des œufs provenant de volailles élevées en plein air. Enfin, la sociabilité s'en trouve également affectée : «Si nous sommes invités, les gens qui nous connaissent bien savent que nous ne mangeons pas de viande et adaptent leur menu, sinon, on mange des salades, il y a toujours quelque chose qui nous convient ", déclare Léa. Marina raconte quant à elle que ses hôtes demandent souvent à l'avance s'il y a des choses quelle ne mange pas : «Quand 


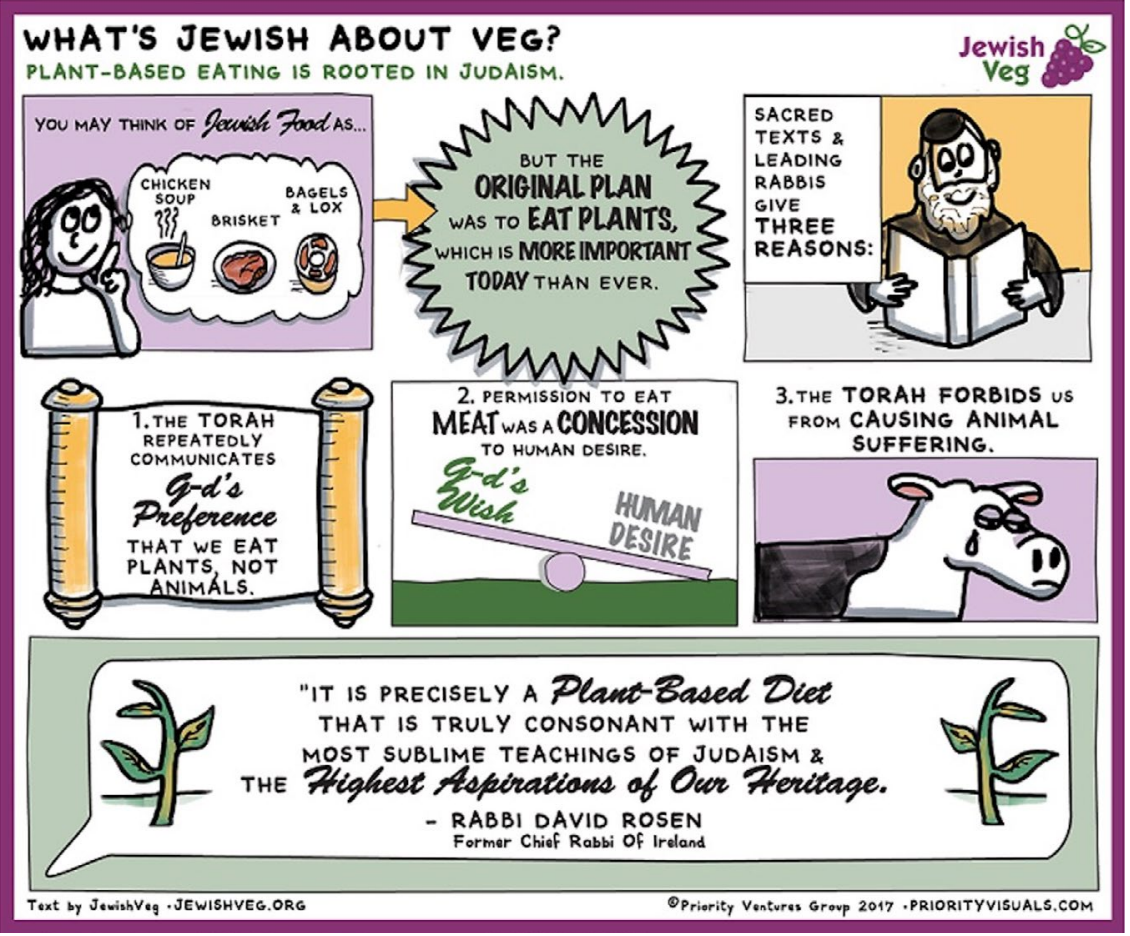

Figure 3 : Illustration du site Internet : les racines du véganisme se trouvent dans la Torah. Source : Jewish Veg, https://www.jewishveg.org/jewish-values-in-action

je dis que je ne mange pas de viande, ni de poulet, je sens que ça embête les gens, mais ils en tiennent compte. Par contre, j'ai un couple de copains qui ne vient pas chez nous le Shabbat si on ne fait pas de viande, même mon mari au début... Aujourd'hui, il s'en passe complètement, d'ailleurs, pour lui, ce qui compte, c'est surtout la question de la protection des animaux. »

Les personnes interrogées ici ne sont pas des militantes de la cause végétarienne, et sont plutôt fléxitariennes. Elles déclarent en général ne pas vouloir imposer aux autres leurs choix alimentaires, ne pas être dans l'extrême ou le dogme, aussi bien en matière de végétarisme que de cacherout. Elles disent toutes leur plaisir de chercher sur Internet, de suivre, d'adapter ou d'inventer de nouvelles recettes de cuisine végane.

Évoquant leur volonté de " progresser » dans cette voie, de « suivre plus la nature ", voire de "faire grandir dans un bout de jardin ou sur une terrasse (leurs) propres légumes », elles s'inscrivent dans un mouvement profond d'évolution des modes d'alimentation et des modes de vie contemporains dans les pays développés. 
Sophie Nizard est socio-anthropologue du judaïsme, chercheur au CéSor (Centre d'études en sciences sociales du religieux-EHESS/CNRS). Son travail de recherche porte sur la construction et la transmission des identités et des mémoires dans le judaïsme contemporain, sur les pratiques et les rites juifs (pratiques alimentaires) et enfin sur la filiation adoptive. Elle a publié de nombreux articles sur ces sujets et un ouvrage sur l'adoption : Adopter et transmettre: Filiations adoptives dans le judaïsme contemporain, Éditions de l'EHESS, coll. En temps \& lieux, 2012. Pour une vue complète de sa bibliographie voir : http://cesor.ehess.fr/2015/03/10/sophie-nizard/

Email : sonizard@hotmail.com

Sophie Nizard is a researcher in sociology and anthropology of Judaism at the CéSor (Center for Social Research on Religion-EHESS/CNRS). She works on the field of Jewish identity and the transmission of Jewish memories in contemporary Judaism. Her interests include the study of food practices in the Jewish tradition (symbolical, ritual, economic, political aspects of the Kosher food), the study of contemporary religious rituals and practices, and an anthropology of adoptive kinship. Nizard has published many articles and a monograph on those subjects: Adopter et transmettre: Filiations adoptives dans le judaïsme contemporain, Éditions de l'EHESS, coll. En temps \& lieux, 2012. (Adopting and Transmitting: Adoptive filiation in Contemporary Judaism). For complete bibliographical data see: http://cesor.ehess.fr/2015/03/10/sophie-nizard/ Email : sonizard@hotmail.com

\section{Notes}

1. La Torah, ou Bible hébraïque, est appelée par les Chrétiens «Ancien Testament». Le mot hébreu Torah signifie Enseignement.

2. Les pastels ou pastillas sont des feuilles de bricks fourrées au poulet ou à la viande. Les borekas sont des feuilletés de pomme de terre, de viande ou de fromage.

3. Le concept de "plat-totem " a été mis en évidence au sujet de l'alimentation en situation de migration et notamment repris par Jean-Pierre Hassoun à propos des pratiques alimentaires des Hmong du Laos en France ; il permet de caractériser des plats qui « voient leur valeur symbolique s'amplifier au point de devenir des marqueurs de la spécificité et de la différence » (Hassoun $1996: 160$ ).

4. La Loi juive relative au Shabbat établit une liste de trente-neuf travaux interdits ce jour-là ; certains sont mentionnés dans le texte biblique comme les travaux des champs ou l'allumage du feu.

5. Extrait d'entretien avec une femme d'origine tunisienne (Nizard 2004 : 96-97).

6. Petits pains frits fourrés de thon, d'œuf dur et de crudités.

7. Omelette de pomme de terre et de thon ou de viande hachée cuite au four. 
8. L'interdit biblique de " cuire le chevreau dans le lait de sa mère » a donné lieu à une interdiction de consommer au cours d'un même repas des laitages et de la viande. Ainsi, aucun repas carné ne pourra être accompagné d'un dessert lacté. C'est pourquoi, nombre de desserts de la cuisine juive ne comportent ni lait, ni beurre, ni crème d'origine animale (Nizard 2007).

9. Sur la fête de Pourim et les échanges rituels de nourritures, voir Nizard (2007 a).

10. Entretien avec une étudiante en biologie, janvier 2020.

11. Talmud de Babylone, Traité Pessahim. Le Talmud est un corpus de textes composé de la Loi orale ou Michna et de ses commentaires ou Guemara. La rédaction du Talmud de Babylone par les rabbins de la Diaspora babylonienne s'est achevée vers la fin du Ve siècle de notre ère.

12. "Et le vin réjouira le cœur de l'homme» (Psaumes 104, 15).

13. Le Temple de Jérusalem construit une première fois par le Roi Salomon selon le Livre des Rois (Xe siècle avant l'ère chrétienne), puis détruit par les Babyloniens (VIe siècle avant l'ère chrétienne) et reconstruit quelques années plus tard, a été détruit une seconde fois par les Romains en 70 de l'ère chrétienne. Les rituels pratiqués au Temple étaient essentiellement des rituels sacrificiels. La consommation de la viande des sacrifices par les prêtres et les sacrifiants y était quotidienne.

14. Entretien avec une jeune femme de 33 ans habitant Jérusalem, avril 2020.

15. Un flexitarien est un mangeur occasionnel de viande. Pour diverses raisons (mais non pour des questions de budget), il évite la consommation de viande, mais fait le choix de la flexibilité.

16. Jonathan Safran Foer, dans son essai " Faut-il manger des animaux? ", développe l'ensemble des arguments devant conduire tout un chacun à devenir végan. Il évoque à plusieurs reprises le gefilte fish de sa grand-mère et ses souvenirs de la dinde de Thanksgiving, tout en décrivant la terrible condition des animaux dans l'Amérique du XXIe siècle (Safran Foer 2011).

17. Substantif du mot cacher, apte en hébreu, la cacherout est l'ensemble des prescriptions et interdits religieux du judaïsme en matière alimentaire. Ces normes sont codifiées dans la Bible hébraïque, le Talmud, et les codes de lois compilés au cours des siècles.

18. Le site Internet Jewish Veg est un exemple de la diversité religieuse et internationale de ce courant. https://www.jewishveg.org/about.

19. En cela, il reprend un argument courant dans la pensée juive contemporaine, notamment avancé par l'écrivain juif américain Isaac Bashevis Singer.

20. L'expression «mesure pour mesure » indique que celui qui commet une faute sera sanctionné par une punition équivalente à (ou relevant du même registre que) la faute commise.

21. Afin d'entériner cette distorsion, lors de la mise à mort d'un animal, le sang sera extirpé de la chair. C'est pourquoi l'abattage rituel (chekhita) consiste à saigner l'animal en un geste rapide et précis. Pour que le geste soit le moins douloureux et le plus rapide possible, le couteau utilisé doit être parfaitement aiguisé et testé avant chaque égorgement. Le chokhet est formé pendant plusieurs années pour être agréé par une autorité religieuse reconnue, c'est-à-dire apte à effectuer ce geste (Nizard 2020).

22. Si l'on exclut la question des ustensiles et de la consommation de vin. En effet, selon les lois de la cacherout, les ustensiles utilisés ne doivent pas avoir été en 
contact avec des aliments non cacher, même s'ils ont été soigneusement nettoyés. Les boissons à base de raisin comme le vin doivent suivre un processus de fabrication spécifique. Par ailleurs, certaines lois agricoles bibliques perdurent dans le monde moderne, comme l'interdit de consommer les fruits d'un arbre durant ses trois premières années de production, l'obligation de prélèvement d'une partie des récoltes ou l'obligation de la jachère tous les sept ans (shmita). Ces lois vont dans le sens d'une limitation du productivisme agricole.

23. Cependant, il est prescrit d'éviter de mélanger viandes et poissons, même si cet évitement ne constitue pas un interdit alimentaire absolu, mais plutôt une habitude qui ne trouve pas de source dans la Bible.

24. C'est l'exemple que prend Jonathan Safran Foer (2011) pour faire comprendre à ses lecteurs la force des habitudes culinaires acquises depuis l'enfance.

25. Le falafel a sans doute été introduit dans le répertoire culinaire israélien par les Juifs du Levant, en particulier du Liban.

26. Pour comparaison, une récente enquête réalisée par le Credoc (2018) estime qu’en France, en plus des 5,2 pour cent de végétariens, végétaliens et végans, 20 pour cent de la population est flexitarienne (Polette de Olivera 2019).

27. Entretien avec une femme de 41 ans habitant Tel Aviv, mars 2020.

28. Entretien avec une femme de 61 ans habitant Jérusalem, réalisé en avril 2020 en pleine crise du Covid-19.

\section{Références}

Abramowitz, Y. (2018), 'La protection de l'environnement, une cause juive', Jerusalem Post, 28 janvier, https://www.jpost.com/Edition-Francaise/Social-Eco/ La-protection-de-lenvironnement-une-cause-juive-540030.

Alfond, K. (2019), The Jewish Food Hero Cookbook (Woodstock: Jewish Lights).

Bahloul, J. (1983), Le culte de la table dressée : Rites et traditions de la table juive algérienne (Paris : Métailé).

Bouanchaud, C. (2018), 'La consommation de viande en France recule depuis 10 ans', Le Monde, 6 septembre, https://www.lemonde.fr/planete/article/2018/09/06/la -consommation-de-viande-en-france-recule-depuis-dix-ans_5350897_3244.html.

Douillet, J. (2018), 'Tsahal, l'armée la plus végétalienne du monde', Alliance, 4 octobre, http://www1.alliancefr.com/actualites/israel-tsahal-armee-vegetalienne-vegan -monde-6070621.

Fargeon, Y. (2013), Le statut des animaux dans la Bible [Vidéo en hébreu], 11 février, YouTube : https://www.youtube.com/watch?v=hSIHegCU_wQ\&feature=youtu.be.

Green, A. (s. d.), Vegetarianism: An Alternative Kashrut, My Jewish Learning, Consulté le 5 janvier 2020 sur https://www.myjewishlearning.com/article/vegetarianism -an-alternative-kashrut/.

Hassoun, J-P. (1996), 'Les pratiques alimentaires des Hmong du Laos en France, « manger moderne » dans une structure ancienne', Ethnologie française 26 : 151-167.

Kanafani-Zahar, A. Mathieu, S. et Nizard, S. (2007), 'À croire et à manger : penser le lien', in $\grave{A}$ croire et à manger : Religions et alimentation, (ed.) A. Kanafani Zahar, S. Mathieu et S. Nizard (Paris : L'Harmattan/AFSR, coll. Religions en questions), 9-16. 
Keissar, A. (2019), Sans Titre [Vidéo en hébreu], 9 juillet, https://www.youtube.com/ watch?v=ba6SJfQaFsg.

Krief, A. (2019), 'Tous les juifs devraient être végétariens', 11 septembre, Jewpop, https://jewpop.com/opinions/tous-les-juifs-devraient-etre-vegetariens/.

Leban, S. (2017), 'Israël : terre promise des végans', Paris Match, 8 août, https://www .parismatch.com/Actu/International/Israel-terre-promise-des-vegans-1322075.

Morin, E. (1984), 'Témoignage', Traces 9-10, “Judaïsme, judaïcité, actes de langage” : 262-269.

Nizard, S. (2020, à paraître), 'Le rapport à l'animal dans la tradition juive', in Religion et écologie, (ed.) C. Andrew Labrecque, (Québec: Presses de l'Université de Laval).

Nizard, S. (2007 a), 'Un festin et trois dons : Échanges de nourritures à Pourim', in $\AA$ croire et à manger : Religions et alimentation, (ed.) A. Kanafani Zahar, S. Mathieu et S. Nizard, (Paris : L'Harmattan/AFSR, coll. Religions en questions), 187-205.

Nizard, S. (2007 b), 'Tu ne cuiras pas le chevreau dans le lait de sa mère', in L'homme, le mangeur, l'animal : Qui nourrit l'autre? (ed.) J-P. Poulain (Paris : Les cahiers de l'OCHA, 12), 112-120.

Nizard, S. (2004), 'Mémoires incorporées : rites et pratiques alimentaires dans le judaïsme contemporain', in La modernité rituelle-Rites politiques et religieux des sociétés modernes, (ed.) E. Dianteill, D. Hervieu-Léger et I. Saint-Martin (Paris : L'Harmattan/AFSR, coll. Religions en questions), 85-99.

Nizard, S. (1997), 'Léconomie du croire: Une anthropologie des pratiques alimentaires juives en modernité' (Thèse de doctorat, Paris, EHESS).

Ottolenghi, Y. [2011] (2015), Plenty : Les recettes végétariennes de Yotam Ottolenghi (Paris : Hachette).

Polette de Oliveira, E. (2019), 'Combien de végétariens en France ?', Porcmag, 4 novembre, https://www.porcmag.com/economie-business/consommation/ combien-de-vegetariens-en-france?sso $=1589323601$.

Safran Foer, J. [2009] (2011), Faut-il manger des animaux? (Paris : Éditions de l'Olivier). Vegan Tel Aviv (s.d.), [page Facebook], Facebook, Consulté le 6 avril 2020 sur https:// www.facebook.com/groups/676307709131516/?ref=pages_profile_groups_tab \&source_id=110511713654246 